\title{
Fast Adaptive Nonuniformity Correction for Infrared Focal-Plane Array Detectors
}

\author{
Esteban Vera \\ Department of Electrical Engineering, University of Concepcion, Casilla 160-C, Correo 3, Concepcion, Chile \\ Email:evera@die.udec.cl
}

\section{Sergio Torres}

Department of Electrical Engineering, University of Concepcion, Casilla 160-C, Correo 3, Concepcion, Chile

Email: storres@die.udec.cl

Received 29 October 2003; Revised 15 January 2005

\begin{abstract}
A novel adaptive scene-based nonuniformity correction technique is presented. The technique simultaneously estimates detector parameters and performs the nonuniformity correction based on the retina-like neural network approach. The proposed method includes the use of an adaptive learning rate rule in the gain and offset parameter estimation process. This learning rate rule, together with a reduction in the averaging window size used for the parameter estimation, may provide an efficient implementation that should increase the original method's scene-based ability to estimate the fixed-pattern noise. The performance of the proposed algorithm is then evaluated with infrared image sequences with simulated and real fixed-pattern noise. The results show a significative faster and more reliable fixed-pattern noise reduction, tracking the parameters drift, and presenting a good adaptability to scene changes and nonuniformity conditions.
\end{abstract}

Keywords and phrases: infrared detectors, focal-plane array, nonuniformity correction, fixed-pattern noise, neural networks, least mean square.

\section{INTRODUCTION}

Infrared detectors are widely used in a variety of applications such as defense, surveillance, remote sensing, and astronomy. Usually, the infrared imaging sensors are based on the infrared focal-plane array (IRFPA) technology $[1,2,3]$. An IRFPA can be considered as an array of independent detectors aligned at the focal plane of the imaging system. Unfortunately, every detector on the IRFPA can have different responses under the same stimulus, what is considered as the nonuniformity problem, leading then to the presence of a fixed-pattern noise (FPN) in the resulting images. Furthermore, this FPN presents a noticeable temporal drift, which is even severe in uncooled infrared systems.

Under this scope, nonuniformity correction (NUC) is a necessary and unavoidable task to be performed in order to achieve higher-quality infrared images, or image sequences, eliminating thus the unwanted FPN. In this way, NUC techniques normally assume a linear model for the detectors, characterizing the nonunifomity response problem as a gain and offset estimation problem per detector.

The most accurate NUC methods are based on the use of uniform infrared sources, where the simplest and most used one is the two-point calibration [4], which employs two blackbodies at different temperatures to calculate the exact gain and offset of each detector on the IRFPA through the use of a simple line fitting procedure. However, these referencebased NUC methods must be often repeated in order to follow the temporal drift of the nonuniformity characteristic parameters, necessarily halting the normal operation of the system to allow the use of the blackbody sources, interrupting the scene anyway.

On the other hand, scene-based NUC techniques only make use of the readout infrared data captured during the normal operation of the imaging system, reducing the optical setup complexity, and avoiding scene interruptions. Nonetheless, the scene-based approach is well known for requiring higher computational resources (memory and MFLOPS), and also for achieving less accurate results compared to the calibration methods. The larger memory requirements are related to the use of block of frames to perform the gain and offset estimation in a consistent way, neglecting the drift on the parameters over the block duration. The accuracy, or nonuniformity correction performance, is up to the algorithm used, the scene variation, and the size of the block of frames previously chosen. That is the case of the constant statistics (CS) method, first developed by Narendra in [5], and lately revisited and enhanced by Harris and 
Chiang [6], where, in spite of its simplicity, the achieved performances are strongly dependent on the scene diversity, or motion, which can only be compensated using larger blocks of data to reinforce the needed statistics. Anyway, and following the same block-based approach, many other efforts are recently being devoted to the development of novel scenebased techniques $[7,8,9,10,11,12]$, taking advantage of advanced image processing techniques apart from the classical statistics, such as motion vectors, image registration, and Wiener and Kalman filtering. However, and regardless of the scene-based NUC technique employed, the intrinsic scenedata dependence is in most of the cases responsible for a noticeable performance degradation, specially when the NUC methods are applied over scenes with lack of motion and low contrasts, which could finally constraint the range of applications where such scene-based methods could be successfully employed. Thus, the main challenge of all these block-based NUC methods is to obtain a reasonable NUC performance using smaller block sizes (fewer data frames), reducing as much as possible the unavoidable scene data dependence.

Then, and also following the constant need of computational resources reduction, in this paper, special attention is given to the adaptive scene-based NUC developed by Scribner et al. $[13,14]$. This method has the ability to estimate the needed gain and offset parameters under a frame by frame basis by means of the least mean square (LMS) algorithm [15], using a retina-like neural network approach. In this way, the nonuniformity parameters are dynamically updated as new image frames arrive, and the nonuniformity correction is simultaneously performed by the same neural network model. With this approach, the algorithm is also able to track the parameters drift while it is occurring. Nonetheless, in spite of its straightforward real-time implementation, and the low computational load required, this method is hard to set up for providing robustness and independence to the information provided by the scene data, sacrificing estimation speed by accuracy or vice versa.

Therefore, seeking for more effectiveness in the use of the scene information, and also exploiting even further the tremendous potential of the original Scribner's method [13], in this paper, an enhanced version of his NUC technique is presented. Specifically, the fast adaptive NUC method here proposed relies on the use of an adaptive learning rate schedule in the parameters estimation update process, based on a custom variation of the normalized least mean square (NMLS) algorithm [15]. This adaptive learning rate is chosen to be dependent on the spatial content of the readout data, constraining thus the speed of the learning process for each detector on the IRFPA independently. In this way, faster adaptation is mainly allowed for such detectors (pixels) where the input data fits the hypothesis assumed by the retinomorphic model, as presented by Scribner in his seminal work [13]. This new degree of adaptiveness added to the Scribner's NUC method may permit a better and faster estimation for the nonuniformity parameters, which in theory may lead to an improved overall NUC performance.

This paper is finally organized as follows. In Section 2, the adaptive NUC technique is presented, and the proposed enhancements developed. In Section 3, the proposed technique is tested and compared using sequences of infrared data with simulated nonuniformity. In Section 4, the technique is analyzed when applied to sequences of real infrared data. The conclusions obtained are summarized in Section 5 .

\section{ADAPTIVE SCENE-BASED NUC METHOD}

The goal of this paper is the development of a novel adaptive scene-based NUC method for reducing the FPN in a fast and reliable frame by frame basis. Then, we start from an avenue introduced by Scribner et al. [13, 14], briefly showing and explaining their retina-like neural net NUC method, and after discussing its weaknesses and drawbacks, we finally motivate the introduction of our proposed innovations.

\subsection{Method description}

First, we assume that each infrared detector is characterized by a linear model. Then, for the $i j$ th detector in the focalplane array, the measured readout signal $Y_{i j}$ at a given time $n$ can be expressed as

$$
Y_{i j}(n)=g_{i j}(n) \cdot X_{i j}(n)+o_{i j}(n),
$$

where $g_{i j}(n)$ and $o_{i j}(n)$ are the gain and the offset of the $i j$ th detector, and $X_{i j}(n)$ is the real incident infrared photon flux collected by the respective detector. As proposed by Scribner, (1) is reordered as follows:

$$
X_{i j}(n)=w_{i j}(n) \cdot Y_{i j}(n)+b_{i j}(n)
$$

where the new parameters $w_{i j}(n)$ and $b_{i j}(n)$ are related to the gain and offset parameters of each detector as follows:

$$
w_{i j}(n)=\frac{1}{g_{i j}(n)}, \quad b_{i j}(n)=-\frac{o_{i j}(n)}{g_{i j}(n)} .
$$

The expression presented in (2) is responsible for performing the nonuniformity correction on the readout data. Then, for each $i j$ th detector, the NUC model (2) can be considered as the simplest neural network structure [16], which consists of a single linear neuron node with a weight $w_{i j}(n)$ and a bias $b_{i j}(n)$. Thus, the readout data $Y_{i j}(n)$ is the input to the $i j$ th neuron, and its output $X_{i j}(n)$ is now the estimation obtained for the real infrared data. The entire array of single-input/single-output linear neurons finally constitutes the neural network structure for the Scribner's adaptive NUC method.

This neural network approach allows the use of linear regression techniques to perform the estimation of each neuron's parameters. To accomplish this estimation task, the needed error function $E_{i j}(n)$ is defined as the difference between a desired target value $T_{i j}(n)$ and the estimated infrared data $X_{i j}(n)(4)$. Moreover, and inspired on an analogy with biological retina-like processes, the target value proposed by Scribner is assumed as the local spatial average, or mean filter, of the output data $X_{i j}(n)$ (5). This assumption is based on the 
retinomorphic hypothesis, which states that there is a higher probability for a given detector, and its surrounding neighbors, of being illuminated by the same infrared irradiance level. Therefore, the error function is finally given by

$$
E_{i j}(n)=T_{i j}(n)-X_{i j}(n)
$$

where the desired target value $T_{i j}(n)$ is calculated as

$$
T_{i j}(n)=\frac{1}{(2 v+1)^{2}} \sum_{k=i-v}^{i+v} \sum_{l=j-v}^{j+v} X_{k l}(n),
$$

and $2 v+1$ is the kernel neighborhood, or averaging window size. As a remark, in Scribner's related work [13, 14], a $21 \times 21$ window $(v=10)$ is chosen as the optimum value for NUC purposes.

Then, in order to minimize the error $E_{i j}(n)$ in the mean square error sense, the functional $J_{i j}$ and its partial derivatives (gradients) related to the desired parameters to be estimated are expressed as follows:

$$
\begin{aligned}
J_{i j}=\sum_{n} E_{i j}(n)^{2} & =\sum_{n}\left(T_{i j}(n)-X_{i j}(n)\right)^{2} \\
\frac{\partial J_{i j}}{\partial w_{i j}} & =-2 \cdot E_{i j} \cdot Y_{i j} \\
\frac{\partial J_{i j}}{\partial b_{i j}} & =-2 \cdot E_{i j} .
\end{aligned}
$$

This functional $J$ is minimized only when its both gradients in (7) are equal to zero. Fortunately, and as proposed by Scribner, this optimization problem can be solved by using the least mean square (LMS) algorithm $[15,16]$. In this gradient-based search algorithm, also known as the steepest descent algorithm, the parameters to be estimated are recursively and smoothly updated with a portion of each respective error gradient, just as follows:

$$
\begin{aligned}
& w_{i j}(n+1)=w_{i j}(n)-\eta \cdot E_{i j}(n) \cdot Y_{i j}(n), \\
& b_{i j}(n+1)=b_{i j}(n)-\eta \cdot E_{i j}(n)
\end{aligned}
$$

where $\eta$ is a fixed parameter known as the learning rate, which has the task of controlling the convergence speed of the algorithm. Normally, larger values for $\eta$ can provide a faster convergence speed, but smaller values can assure better stability instead. However, as the learning rate $\eta$ is a global parameter for the whole NUC neural network structure, its chosen value can only be as fast (or large) as more confident is each error measure $E_{i j}(n)$ for all the $i j$ th neurons at every time step $n$.

Specifically in the NUC sense, the mentioned error confidence is directly dependent on how accurate is the estimated target value $T_{i j}(n)$ compared to the real incident photon flux collected by each single detector. Hence, the final responsibility relies on the validity of the retinomorphic hypothesis and the proper choice of its associated target function.
Nonetheless, the estimated target value for each neuron is strongly dependent on the scene dynamics and the scene itself, affecting not only the accomplishment of the retinomorphic assumption, but also the error confidence for the overall adaptive NUC structure during the continuous adaptive estimation process. As a consequence, very small learning rate values are commonly used, leading to a very slow, but safe, convergence.

\subsection{Fast adaptive NUC development}

The latest discussion about the learning rate parameter $\eta$, and its incidence on the speed and robustness behavior of the NUC algorithm, is the selected starting point for the development of the proposed adaptive learning rate rule. The main idea behind the use of an adaptive learning rate is to increase the efficiency of the learning process, which in the adaptive NUC case would help not only in speeding up the FPN reduction, but also in decreasing the scene data dependence, finally improving the overall NUC performance.

As mentioned before, it is clear that the error measure is more confident when the target value is as close as possible to the real incident infrared irradiance. But following the retinomorphic assumption, when could the target function perform in such a good way? Well, an accurate behavior of the target function can only be assured when the spatial content of a given pixel and its surroundings is as similar as possible, hopefully only containing spatial noise generated by the nonuniformity instead of some useful scene information. In this way, it can be guaranteed that when a spatial mean filter is used, the calculated value should be a very good spatial noiseless estimation for that neuron output, so a large learning rate value could be safely used. On the other hand, when the spatial content of a pixel neighborhood is too spread, there are more chances of deviating the target value from the real desired one, which in the adaptive NUC sense is equivalent to say that the retinomorphic hypothesis is not fulfilled, and therefore a small value for the learning rate should be used.

Then, as a basis for developing the dynamic variation of the adaptive learning rate to be used, we selected the normalized least mean square algorithm (NLMS) [15] in replacement of the LMS. In this algorithm, $\eta$ is no longer a fixed number, but a variable one calculated as $\eta(n)=\mu /\left(\|\mathrm{x}(n)\|_{2}^{2}+\right.$ $\epsilon)$, where $\mu$ is a constant that fix the maximum allowable value for $\eta,\|\mathrm{x}(n)\|_{2}$ denotes the Euclidean norm of the input vector $\mathrm{x}$ at a given time $n$, and $\epsilon$ is a small positive constant used for stability.

Assuming that the adaptive NUC scheme consists in an array of single neurons with separated parameter estimation processes, we propose the use of an independent adaptive learning rate $\eta_{i j}(n)$ for each $i j$ th neuron in the adaptive NUC neural net structure. Moreover, in order to adapt the NLMS to every $\eta_{i j}(n)$, we propose the use of the local spatial standard deviation of the input image $\sigma_{Y_{i j}(n)}$ as a measure of distance, replacing the Euclidean norm formerly used in the original NLMS algorithm. But in order to reinforce even further the retinomorphic assumption, we also propose that the input vector for each $\sigma_{Y_{i j}(n)}$ must consist in the 


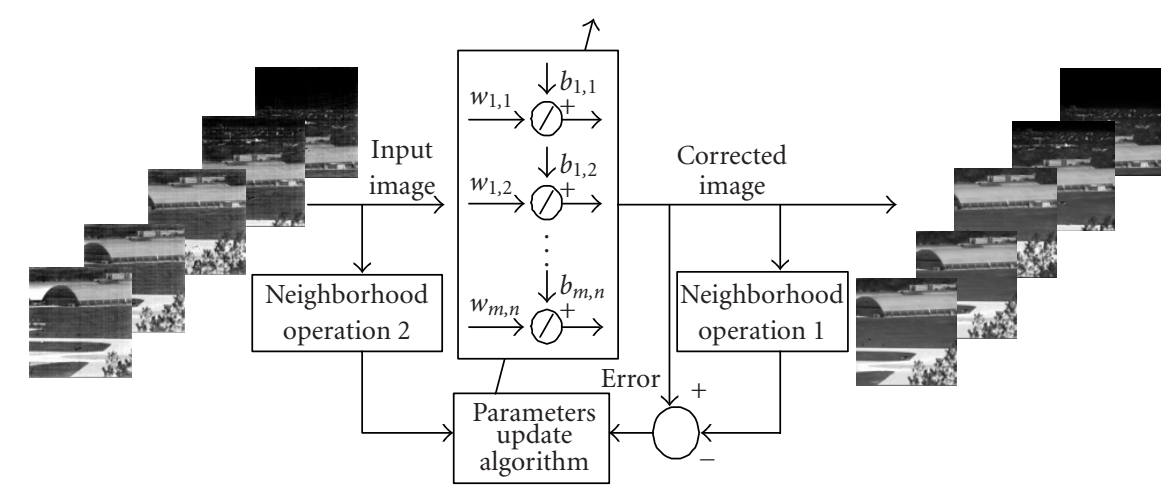

FIGURE 1: Scheme of the proposed fast adaptive scene-based nonuniformity correction method.

TABLE 1: Statistical parameters to generate the simulated fixedpattern noise test image sequences.

\begin{tabular}{lcccccc}
\hline & $\bar{g}$ & $\sigma_{g}$ & $\bar{o}$ & $\sigma_{o}$ & $\bar{t}$ & $\sigma_{t}$ \\
\hline Test sequence 1 & 1 & 0.025 & 0 & $5 \%$ & 0 & $0.5 \%$ \\
Test sequence 2 & 1 & 0.05 & 0 & $10 \%$ & 0 & $0.5 \%$ \\
\hline
\end{tabular}

same neighborhood used for the desired target $T_{i j}(n)$ calculation. In this way, $\sigma_{Y_{i j}(n)}$ becomes a confidence measure of the ability of $T_{i j}(n)$ to fit the retinomorphic hypothesis. Thus, the adaptive learning rate rule designed for the fast adaptive NUC algorithm here proposed is expressed as follows:

$$
\eta_{i j}(n)=\frac{k_{\mathrm{alr}}}{1+\sigma_{Y_{i j}(n)}}
$$

where $\epsilon=1$ and $\mu=k_{\text {alr }}$ in respect to the NLMS algorithm. Thereby, if a given piece of the input image (a pixel and its neighbors) is smooth enough, then the desired averaged target value at the output is more confident, and the learning rate assumes larger values. On the other hand, if the local input standard deviation in the surroundings of a certain pixel is too high, like in an object border, the correspondent learning rate assumes smaller values. Thus, in order to add this adaptive learning rate to the NUC algorithm, $\eta$ in (8) must be replaced by its counterpart $\eta_{i j}(n)$.

The overall fast adaptive NUC scheme is presented in Figure 1, where the input image is the readout data $Y_{i j}(n)$, which enters the adaptive NUC neural net model (represented by an array of linear neurons) that calculates the corrected image version $X_{i j}(n)$. The local spatial average function (neighborhood operation 1) is applied to the estimated corrected image $X_{i j}(n)$, generating thus the target $T_{i j}(n)$ in order to calculate the error function $E_{i j}(n)$. The error is then needed in the steepest descent parameters update algorithm, where the adaptive learning rate $\eta_{i j}(n)$ utilizes the local spatial standard deviation (neighborhood operation 2) of the input image.

\section{NONUNIFORMITY CORRECTION WITH SIMULATED DATA}

Several tests were performed over data sequences with simulated FPN. The first test was designed for checking the influence of different averaging window sizes on the adaptive NUC performance. The second one was designed for studying the adaptive NUC behavior under different learning rate values. Finally, the simulated noisy sequences were used to test the proposed method with the adaptive learning rate. The metric used to measure the NUC performance is given by the peak signal-to-noise ratio (PSNR), which is widely used in the image processing literature to quantify the differences between two images, and it is defined as

$$
\mathrm{PSNR}=20 \log _{10}\left(\frac{p}{\mathrm{RMSE}}\right)
$$

where $p$ is the largest possible value for a single pixel (in this case $p=2^{16}-1$ for 16-bit images), and RMSE is the root mean square error of the difference between two images. The PSNR is finally given in decibel units $(\mathrm{dB})$, and it is used throughout this paper to measure the overall difference between a clean reference image against its noisy and nonuniformity corrected versions.

\subsection{Simulated data preparation}

A previously calibrated infrared data sequence with 4000 frames was used to produce image sequences with simulated nonuniformity. The sequence originally contained scene motion introduced by moving the camera and by also recording some moving targets. Two levels of nonuniformity were chosen to generate the fixed gain and offset image masks to produce the desired fixed-pattern noise appearance over each sequence. The selected values for the mean of the gain $(\bar{g})$ and offset $(\bar{o})$, and for the standard deviation of the gain $\left(\sigma_{g}\right)$ and offset $\left(\sigma_{o}\right)$, are displayed in Table 1. In addition, a small temporal noise component, with mean $\bar{t}$ and standard deviation $\sigma_{t}$, was finally added on both synthetic sequences to simulate the common electronic/thermal noise. 


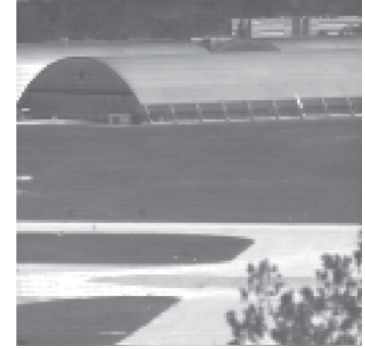

(a)

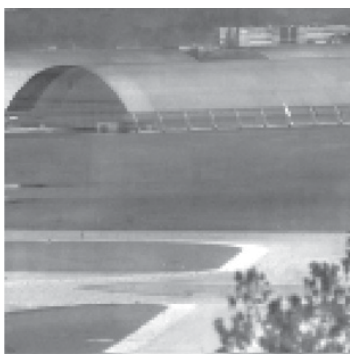

(c)

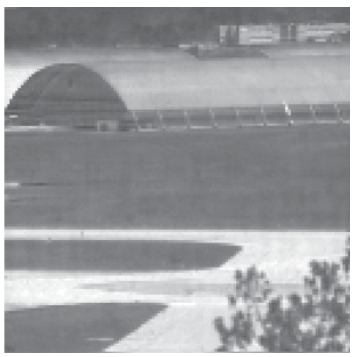

(e)

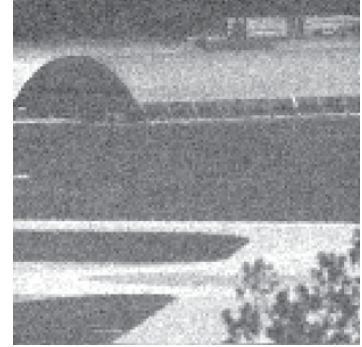

(b)

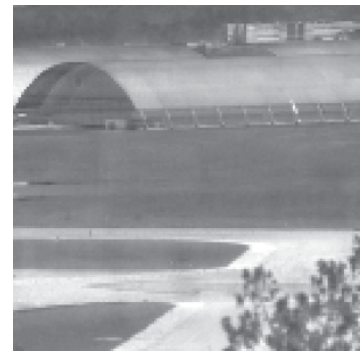

(d)

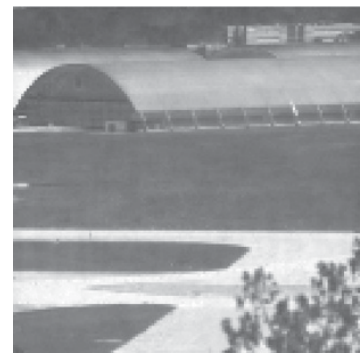

(f)
Figure 2: Frame 1602 of the following. (a) Original sequence. (b) Noisy test sequence 1 (PSNR $=26 \mathrm{~dB})$. (c) NUC with $\eta=0.005$ and a $21 \times 21$ averaging window $(P S N R=21.14 \mathrm{~dB})$. (d) NUC with $\eta=$ 0.005 and a $9 \times 9$ averaging window $(P S N R=24.78 \mathrm{~dB})$. (e) NUC with $\eta=0.005$ and a $3 \times 3$ averaging window $(P S N R=31.60 \mathrm{~dB})$. (f) NUC with a $3 \times 3$ averaging window and an adaptive learning rate using $k_{\text {alr }}=0.075(\mathrm{PSNR}=35.07 \mathrm{~dB})$.

The generation of the test sequence 1 was based on the statistics of real-life measurements on typical IRFPA systems. On the other hand, test sequence 2 was generated with the double of the spatial noise over the gain and offset, just for testing the ability of the methods under stronger FPN conditions. In this way, each synthetic noisy sequence achieved a constant image quality of $26 \mathrm{~dB}$ and $20 \mathrm{~dB}$, respectively, over their whole length. An image sample of the original clean sequence and its noisy counterpart in test sequence 1 are shown in Figures $2 \mathrm{a}$ and $2 \mathrm{~b}$, respectively.

Finally, and in order to perform fair comparisons, all simulated NUC techniques under analysis have been initialized with an unitary gain and null offset. Then, the first output frame is exactly the same as the noisy input one, forcing the same PSNR value as the starting point for the quality measure of all the adaptive NUC methods to be tested.

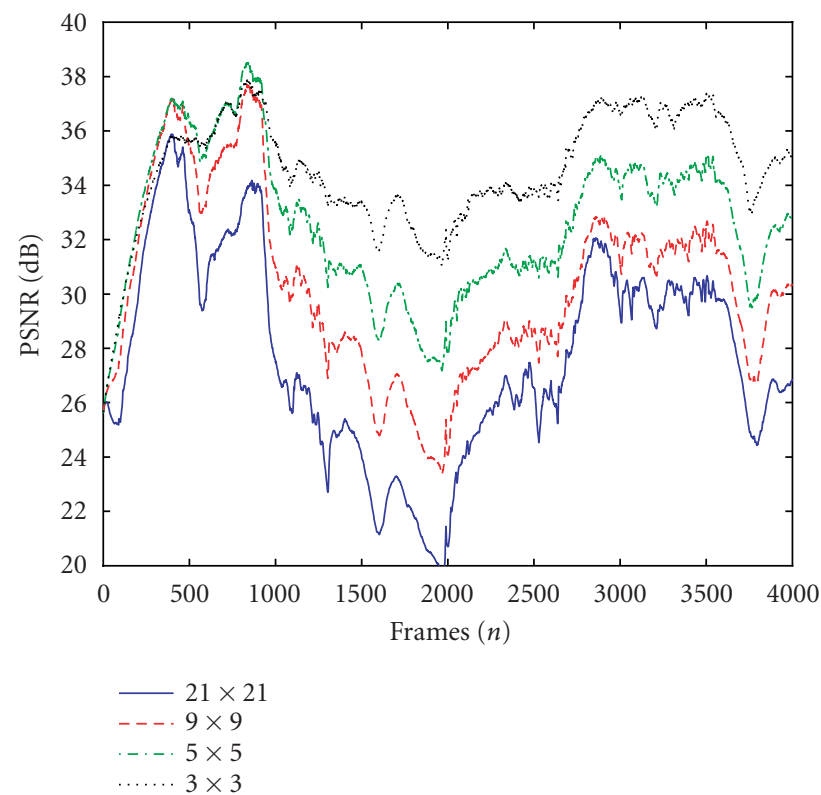

FIGURE 3: Comparison of the adaptive NUC performance obtained with different averaging window sizes when correcting the synthetic $26 \mathrm{~dB}$ noisy test sequence using the same learning rate $\eta=0.005$.

\subsection{Averaging window size analysis}

The first experiment was designed in order to test the adaptive NUC performance when the averaging window size, used to calculate the desired target output, is changed. In addition, the experiment is repeated for several learning rate values, checking the degree of relationship between both adaptive NUC parameters.

As an example, the performance chart over the whole test data sequence, corrected using $\eta=0.005$, can be observed in Figure 3, where it is noticeable that for larger window sizes the performance is worst than the one achieved with smaller values, and it is even noisier than the original $26 \mathrm{~dB}$ level of the test sequence. In this particular case, the performance of the $3 \times 3$ window is practically never surpassed over the whole sequence length, and it looks more stable, never going behind the $32 \mathrm{~dB}$ quality.

This can be verified by checking the corrected frame samples presented in Figures $2 \mathrm{c}, 2 \mathrm{~d}$, and 2e. In that particular frame, a noticeable amount of ghosting-like artifacts can be seen, like a piece of the hangar at another position in a previous frame, appearing over the corrected scene with the $21 \times 21$ window. It can also be noticed that these artifacts are being reduced as the window size decreases. Such kind of behavior can usually happen after even a brief stop on the scene motion, showing us that the algorithm is very sensitive to the quality of the target estimation, and to the scene motion itself. In the particular case when larger window sizes are used, the estimation is clearly more distorted for the gain and offset parameters, moving them away from the real nonuniformity parameters of the IRFPA, generating a new kind of FPN finally reflected as ghosting artifacts. Then, the use of smaller averaging windows looks more robust to the lack of 
TABLE 2: Mean PSNR for the adaptive NUC sequences obtained with different averaging window sizes and learning rate values over the synthetic noisy test sequences.

\begin{tabular}{c|ccc|ccc}
\hline Noise level & \multicolumn{3}{|c|}{$26 \mathrm{~dB}$} & \multicolumn{3}{c}{$20 \mathrm{~dB}$} \\
\cline { 2 - 7 }$\eta$ & 0.005 & 0.0025 & 0.001 & 0.005 & 0.0025 & 28.001 \\
\hline $21 \times 21$ & 27.6821 & 29.4850 & 31.1547 & 26.8354 & 28.4838 & 29.9704 \\
$9 \times 9$ & 30.2413 & 32.1126 & 33.3474 & 30.9378 & 31.0739 & 29.8505 \\
$5 \times 5$ & 32.5977 & 34.0924 & 34.4145 & 30.6324 & 30.9352 & 28.6677 \\
$3 \times 3$ & 34.5585 & 35.1503 & 33.9829 & 31.5127 & \\
\hline
\end{tabular}

scene motion, reducing the blurring that the averaging filter will continuously produce to a static scene.

The results of the mean PSNR value for each corrected sequence are summarized in Table 2, for all the window sizes and learning rates tested. From the table it can be checked that for both noisy sequences $(26 \mathrm{~dB}$ and $20 \mathrm{~dB})$, the $3 \times 3$ window size have obtained the best overall performance, achieving $9 \mathrm{~dB}$ and $11 \mathrm{~dB}$ of respective quality enhancement. Another relevant issue to be noted when using larger window sizes is that better performances can be achieved with lower learning rates. On the other hand, as the window size decreases, a slight increase on the learning rates can be used to enhance the performance. For the highest used learning rate, the best performance is achieved by the smaller window in both cases, and for the lower learning rate value the best performance moves to the $5 \times 5$ one, which finally indicates that smaller window sizes allow the use of faster learning rates.

The clear advantage on the performance issue when the $3 \times 3$ window size is used can be explained with the retinomorphic model assumption, specially if the FPN level is under normal levels. It is more probable that a pixel and its neighbors could receive the same amount of infrared photon flux inside a smaller region, such as a $3 \times 3$ one, than in larger regions such as the $21 \times 21$. Then, smaller windows are better in order to guarantee that the calculated target value is more confident. Then, apart from adding extra complexity to the adaptive NUC method, the use of larger averaging windows is not justified, at least in the case of normal fixed-pattern noise levels.

\subsection{Learning rate analysis}

During the previous analysis, it was shown that the use of smaller window sizes, such as the $3 \times 3$ one, is recommendable to achieve higher NUC performances. Then, the learning rate analysis will be specially focused in this case. In this way, the comparison can be performed between corrections obtained under a very similar quality of the error calculation, or the desired target estimation, due to the use of the same retinomorphic function.

Therefore, if the conditions of the scene data lead to a bad estimation of the target values, and thus in the gain and offset parameters, then an increase of the learning rate will only produce a fast decrease on the performance until the quality of the estimation could be improved, which could only happen if the scene data allows it. This kind of idea can be retrieved from the results obtained using three different learning rate values, which are displayed in Figure 4.

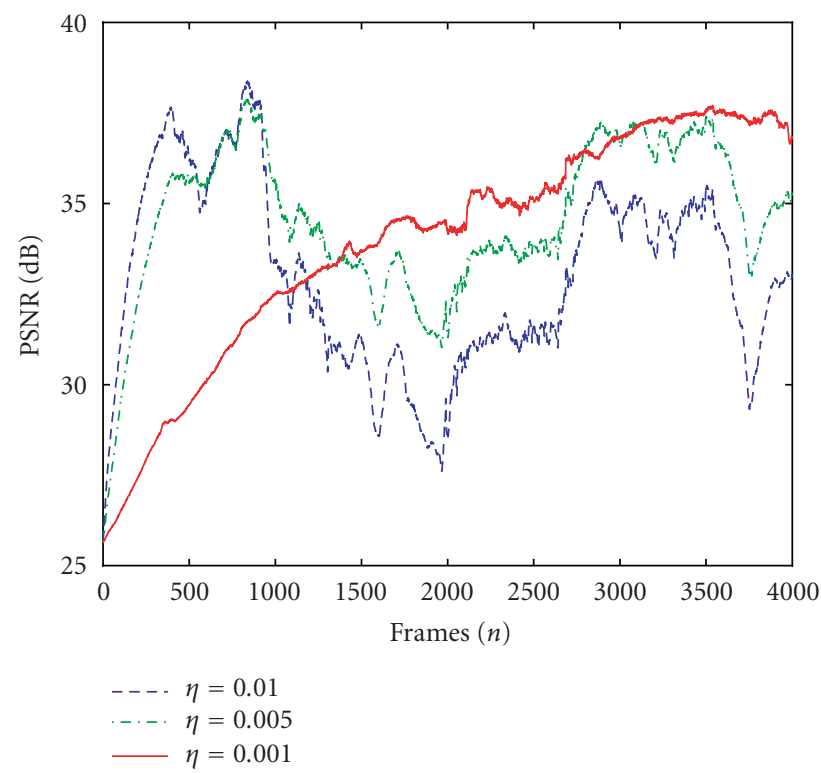

FIGURE 4: PSNR results of the synthetic $26 \mathrm{~dB}$ noisy test sequence corrected using different learning rates and the same averaging window of $3 \times 3$.

From the figure, it is observed that smaller learning rates have slower and softer performance curves, but much more stable than for larger values. In this case, the time needed to adapt to the unknown FPN conditions is extremely large compared with the use of bigger values. Using $\eta=0.01$ only takes 300 frames to cross the $35 \mathrm{~dB}$ barrier, compared to the 2000 frames needed by the $\eta=0.001$. Nonetheless, from the graphs it can be seen that increasing the learning rate can have the same effect of increasing the averaging window, showing strongest deviations on the quality measurement, reducing thus the robustness. However, such observation cannot be sustained if only the overall values displayed in the last row of Table 2 are seen, where with $\eta=0.005$ the performance is $0.5 \mathrm{~dB}$ over $\eta=0.001$, which may be reversed if only the last half of the results are used to calculate the average PSNR, just when the slower learning rate is starting to reach a good performance level.

With the use of a fixed learning rate, the NUC algorithm is not able to know when the error is good or not to perform an accurate parameter update towards the nonuniformity correction, weighting in the same way when favorable or not so favorable conditions are present, specially in some areas 


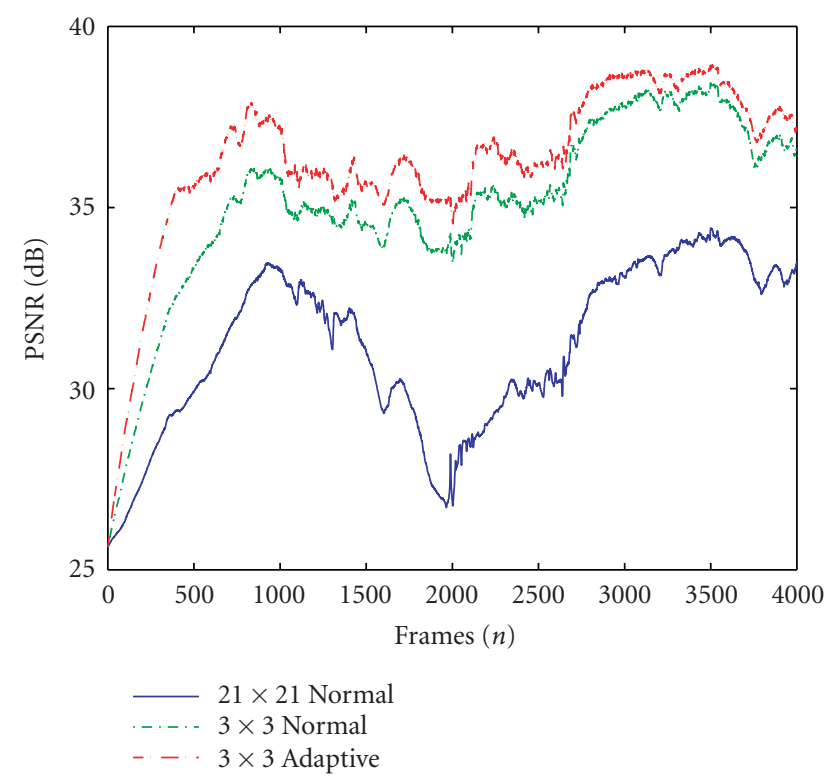

FIGURE 5: PSNR results of the synthetic $26 \mathrm{~dB}$ noisy test sequence corrected using a $21 \times 21$ averaging window with $\eta=0.001$, a $3 \times 3$ averaging window with $\eta=0.0025$, and a $3 \times 3$ averaging window with adaptive learning rate of $k_{\mathrm{alr}}=0.075$.

of the input images, which finally constraints the maximum value for the learning rate to be used. In addition, smaller values lead to a very slow achievement of acceptable results. Therefore, a good balance between correction quality, convergence speed, stability, and scene data dependence must be considered in order to guarantee a successful use of the adaptive NUC method, choosing the proper learning rate for each situation.

\subsection{Adaptive learning rate analysis}

From both previous analyses, the learning rates which have provided the best results with the $21 \times 21$ averaging window proposed by Scribner, and also with the $3 \times 3$ reduced window size, are the adaptive NUC parameters selected to perform the comparisons against the use of the proposed adaptive learning rate. To apply the adaptive learning rate, the $3 \times 3$ averaging window is used, mainly because as it was shown in the previous results too, it allows better use of higher learning rate values, which is associated with the wanted speed up of the algorithm, one of the objectives for the proposed new method.

In Figure 5, the performance chart comparison over the whole length of the corrected $26 \mathrm{~dB}$ noisy sequence is presented. Again, the improvement achieved by only reducing the averaging window size is noticeable. In addition, the constant PSNR improvement over the whole corrected sequence provided by the proposed algorithm with the adaptive learning rate is also clear, which can also be checked with the sample frame presented in Figure $2 \mathrm{f}$.

Moreover, from Table 3, it is shown that the best overall performance achieved was of $36 \mathrm{~dB}$ and $32 \mathrm{~dB}$ approximately
TABle 3: Mean PSNR for the fast adaptive NUC applied to the synthetic noisy test sequences.

\begin{tabular}{lcc}
\hline Noise level & $26 \mathrm{~dB}$ & $20 \mathrm{~dB}$ \\
\hline$k_{\mathrm{alr}}=0.075$ & 36.3050 & 31.2221 \\
$k_{\mathrm{alr}}=0.125$ & 35.6729 & 32.0483 \\
\hline
\end{tabular}

for both $26 \mathrm{~dB}$ and $20 \mathrm{~dB}$ noisy test sequences, which is $1 \mathrm{~dB}$ above the best corrections obtained with fixed learning rates. These results confirm the improvement on the quality when the adaptive learning rate is used. Nonetheless, it must be recalled that the value for $k_{\text {alr }}$ must be increased as the FPN level also increases, because as the standard deviation of the input image logically increases too, if $k_{\text {alr }}$ is maintained, the effective maximum learning rate applied decreases, thus reducing the allowed gain on speed of the algorithm. Anyway, with the NUC quality and the satisfactory convergence speed achieved, plus the stability shown, it looks that the fast adaptive NUC method can really grant the needed robustness to the Scribner's original algorithm.

\section{NONUNIFORMITY CORRECTION WITH REAL INFRARED DATA}

In this section, apart from using real infrared data, the idea is to simulate the behavior of the adaptive NUC algorithms under real operational conditions. For that reason, a special experiment was designed for testing the reduction of the FPN, and thus the ability for estimating the gain and offset parameters, checking also the adaptability to scene changes and the tracking ability to the real parameters drift. Again, the chosen adaptive NUC method parameters are the ones which have reached the best performances on the previous simulations: $21 \times 21$ averaging window with $\eta=0.001,3 \times 3$ averaging window with $\eta=0.0025$, and finally the adaptive learning rate version with a $3 \times 3$ averaging window and $k_{\text {alr }}=0.075$.

\subsection{Real infrared data}

A set of real infrared image sequences has been collected using a $128 \times 128$ InSb FPA camera (Amber Model AE-4128), operating in the $3-5 \mu \mathrm{m}$ range, and working at 30 frames per second. In this way, five 4000-frame data sequences were taken sequentially at 6:30 AM, 8:00AM, 9:30AM, 11:00AM, and 1:00PM, during the same day. Moreover, an additional data sequence was taken at an unknown time with the same camera.

\subsection{Experiment design}

The proposed experiment is based on a simulation of the turn-on and turn-off operation of a real camera, taking advantage on the variety of the real infrared data available. In this way, the experiment is useful to check the adaptability of the different algorithms under furtive and unexpected changes in the scene, and also their abilities to estimate quickly or not the unknown FPN and its drift.

In order to perform the intended experiment, subblocks of 400 frames of each one of the 6 available real IR sequences 
were selected and then merged in a unique long sequence, keeping the chronological order, but placing the unknown time subsequence before the last subsequence of the 1:00PM. Then, the final 2400-frame sequence of real infrared data is corrected by the adaptive NUC algorithms under test, including the proposed new version. Therefore, as the NUC algorithms are applied, the last calculated parameters for each subsequence seem to be virtually stored (they are always stored from any given frame to the next one), simulating thus when the camera is turned off. These stored parameters are obviously used then as the initial conditions for the gain and offset of the next data subsequence to be corrected, simulating this time when the camera is turned on.

\subsection{Performance analysis}

Unfortunately, when dealing with real infrared images, it is not always possible to have, or obtain, the calibration data needed all the time to perform a radiometrically accurate correction to be used as a reference for comparison purposes, so the PSNR cannot be calculated. However, the roughness index $\rho$ is often used in the literature $[11,12]$ as a measure or indicator of the amount of fixed-pattern noise present in a real image, which could also be understood as the degree of nonuniformity on the detector's parameters. The index is calculated as follows:

$$
\rho=\frac{\left\|h_{1} * I\right\|_{1}+\left\|h_{2} * I\right\|_{1}}{\|I\|_{1}}
$$

where $h_{1}$ and $h_{2}$ are a horizontal and vertical difference filter, respectively, $I$ is the image under analysis, $\|f\|_{1}$ is the $l^{1}$ norm of $f$, and $*$ represents discrete convolution. Specifically, the lower the value for $\rho$, the less the fixed-pattern noise is and vice versa.

After the nonuniformity correction over the whole test sequence was done using the selected algorithms, the roughness index $\rho$ was measured for the corrected sequences, and also for the original noisy sequence as a reference for establishing the effective FPN reduction. Results of the performance obtained are presented in Figure 6.

When checking the roughness on the real infrared data sequences (original noisy), it is possible to appreciate the stepwise behavior of the FPN level presented on the transition between the different scenes, or subsequences. As a matter of fact, the nonuniformity level shows a progressive increase as the time of the capture advances too (between subsequence 1 and 4). That happens due to the increase of contrast as the daylight becomes stronger (more infrared irradiance), enlarging then the usage of the dynamic range of the system, which also helps in amplifying the FPN level, demonstrating thus the effect of the gain nonuniformity. Then, it can also be inferred that the subsequence 5 , taken at an unknown time, was maybe captured at night.

Now evaluating the NUC results, it can be seen that when the scenes are presented in a sequential order (subsequences 1 to 4 ), the stored parameters between the scene transitions seem to be quite useful for continuing estimating the FPN. The distances between the roughness achieved by

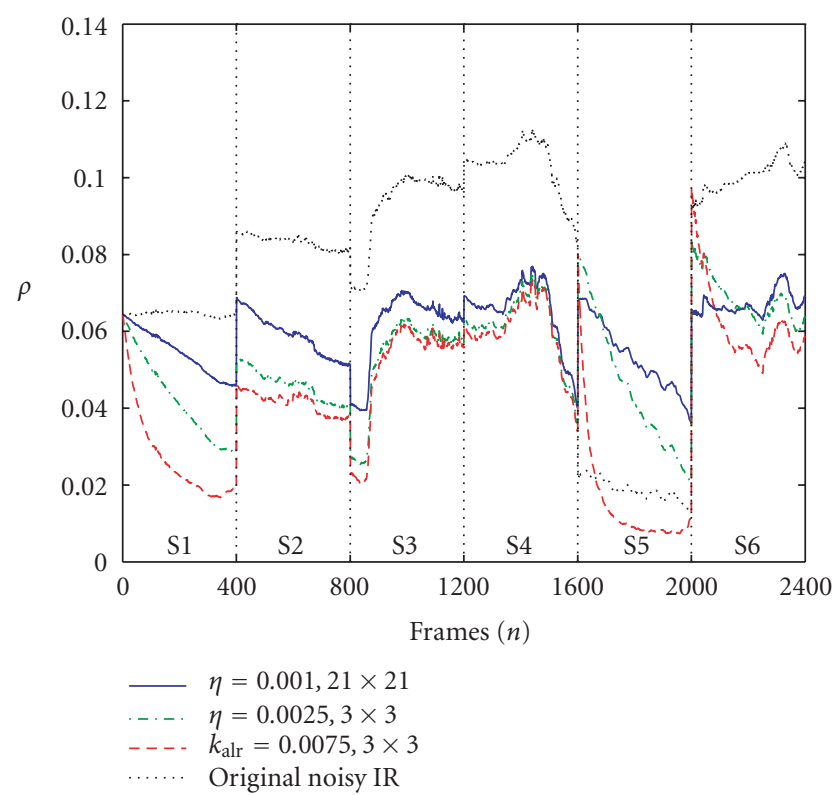

FIGURE 6: Roughness index $\rho$ for the turn-on/turn-off experiment using 6 subblocks of real infrared data sequences (S1-S6). Comparison between the original noisy data and different corrected versions obtained with the adaptive and the proposed fast adaptive NUC method.

all the NUC variations are decreasing as the frame number advances, finally merging at the end of the fourth sequence, reaching a similar level of FPN, and producing very similar visual results at that point. This indicates that the drift of the parameters is very small between scenes taken the same day.

On the other hand, when subsequence 5 (frames between 1601 and 2000) is presented to the NUC algorithms, it seems that the real gain and offset parameters for that sequence were completely different from the ones that the algorithms have estimated successfully using the previous subsequences. Special attention may be given to the roughness of the first frame (1601), where the values for the NUC methods surpassed surprisingly the original FPN of the noisy scene itself. In this way, the nonuniformity parameters previously calculated for the scene subsequence 4 , which have already achieved a good NUC performance, suddenly become completely useless as starting point for the next subsequence. The same situation happens again with the next scene transition (subsequences 5 to 6), because as the parameters are adapting again to the newer unknown nonuniformity conditions of subsequence 5, they are also moving away from the expected NUC parameters for the 1:00PM subsequence, which would logically be closer to the ones already estimated at the end of subsequence 4 .

From the same graph of Figure 6, and during the first subsequence (frames 1 to 400 ), it can be noticed that while all the normal adaptive NUC methods have almost a linear decreasing behavior, an important exponential decrement of the roughness was obtained with the adaptive learning rate version. Next, and during the following subsequences 2, 3, and 4, the fast adaptive NUC just maintains its performance 
TABLE 4: Mean roughness $\rho$ for the adaptive and fast adaptive NUC versions of the real infrared test sequences.

\begin{tabular}{|c|c|c|c|c|c|c|c|}
\hline \multirow{2}{*}{ Sequence number } & \multicolumn{7}{|c|}{$\rho$} \\
\hline & 1 & 2 & 3 & 4 & 5 & 6 & $\overline{1-6}$ \\
\hline Original noisy IR & 0.0646 & 0.0833 & 0.0928 & 0.1031 & 0.0188 & 0.1003 & 0.0771 \\
\hline$\eta=0.001,21 \times 21$ & 0.0545 & 0.0590 & 0.0613 & 0.0655 & 0.0535 & 0.0672 & 0.0601 \\
\hline$\eta=0.0025,3 \times 3$ & 0.0422 & 0.0460 & 0.0529 & 0.0617 & 0.0452 & 0.0683 & 0.0527 \\
\hline$k_{\mathrm{alr}}=0.075,3 \times 3$ & 0.0269 & 0.0414 & 0.0505 & 0.0599 & 0.0150 & 0.0617 & 0.0426 \\
\hline
\end{tabular}

around less than a half of the roughness of the original noisy data, while the other algorithms start to approximate such performance every sequence until all of them finally achieve a similar performance near the frame 1600. Continuing with subsequence 5, although all the NUC algorithms start from a very high roughness value (almost 4 times the original noisy roughness), the adaptive learning rate version provides again a very fast reduction on the roughness, just like it happened in subsequence 1 , but this time even quicker. In this case, the fast adaptive NUC version crosses the original noisy roughness after less than 60 frames ( 2 seconds) of the subsequence, and it also reaches half of the original noisy roughness before the end of the same sub-sequence, while the other algorithms are still over the original roughness level.

Additionally, results of the mean roughness over each sequence are presented in Table 4 . Again, the fast adaptive NUC version here proposed achieves the best roughness index (smaller) over all the subsequences, being the best algorithm in the fixed-pattern noise reduction sense, followed by the constant learning rate with the $3 \times 3$ averaging window, and by the $21 \times 21$ window size proposed by Scribner.

The latest evaluation is even clear when the video sequences generated with the data are visually analyzed. Frame samples at different stages of the nonuniformity correction process developed over the experiment are presented in Figures 7,8 , and 9 .

All of the frame samples shown correspond to the frame 150 of each individual subsequence. In Figure 7, results at the very early scene of the 6:30 AM are presented, where the proposed adaptive learning rate algorithm almost eliminated the FPN after only 5 seconds of the video scene (150 frames, at $30 \mathrm{fps}$ ). However, in Figure 8, related to the third subsequence, the visually achieved FPN level is very similar between all the adaptive NUC versions, which is also confirmed by the roughness value. Finally, both adaptive NUC methods using constant learning rate in Figure 9 were unable, after 150 frames, to adapt to the new FPN of the given fifth subsequence, presenting even more spatial noise than the original image to be cleaned. But with the adaptive learning rate, the spatial noise seems to be already partially removed.

\section{CONCLUSIONS}

In this paper we presented a new scene-based adaptive nonuniformity correction method, proposing a novel way to speed up in a robust way the ability of the original algorithm to reduce the undesired fixed-pattern noise, typically produced by the infrared focal-plane array detectors. It was

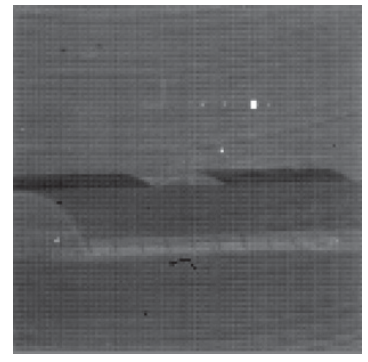

(a)

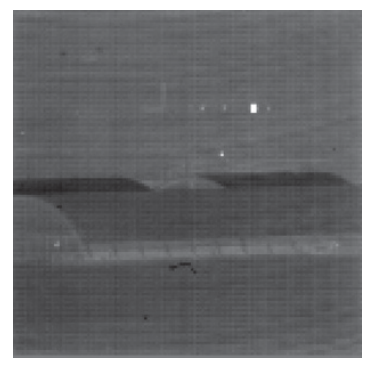

(c)

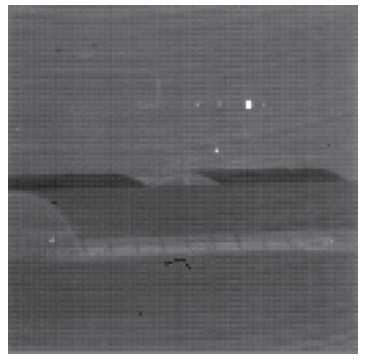

(b)

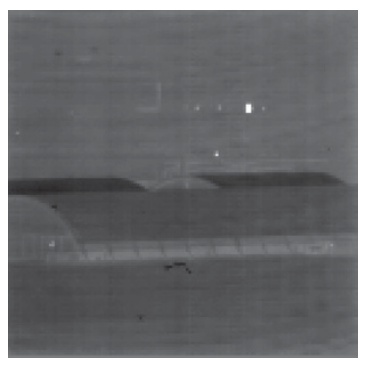

(d)
Figure 7: Adaptive NUC results on real infrared data sequence taken at 6:30 AM. (a) Real infrared data $(\rho=0.0646)$. (b) NUC with $\eta=0.001$ and a $21 \times 21$ averaging window $(\rho=0.0545)$. (c) NUC with $\eta=0.0025$ and a $3 \times 3$ averaging window $(\rho=0.0422)$. (d) NUC with a $3 \times 3$ averaging window with adaptive learning rate using $k_{\text {alr }}=0.075(\rho=0.0359)$.

shown through the simulations that the performance of the adaptive NUC algorithm can be improved drastically by using smaller averaging window sizes, at least for normal spatial noise levels. This smaller windows, used for estimating the gain and offset parameters for the detectors, allowed the use of larger learning rate values, which means faster adaptability but less robustness. Nevertheless, the introduction of the proposed adaptive learning rate rule permitted a safe increase in the effective learning rate used, speeding up the achievement of high-performance nonuniformity corrections, avoiding also the formation of the undesired ghosting artifacts. After several tests under real operational conditions using real infrared data, our proposed fast adaptive NUC method showed the best performance in reducing quickly the fixed-pattern noise, adapting also to abrupt scene changes and different nonuniformity conditions. Anyway, further research may include studies on the use of additional 


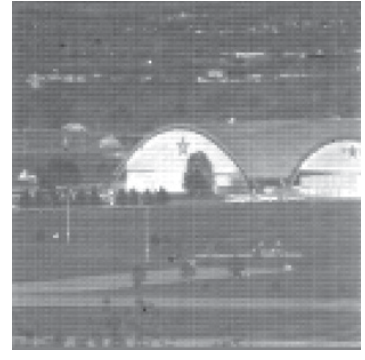

(a)

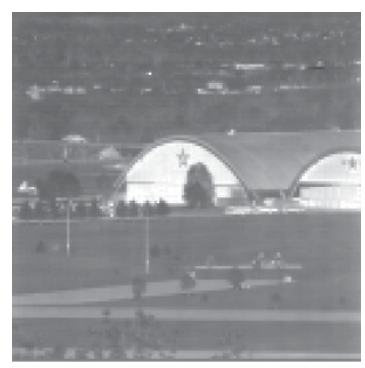

(c)

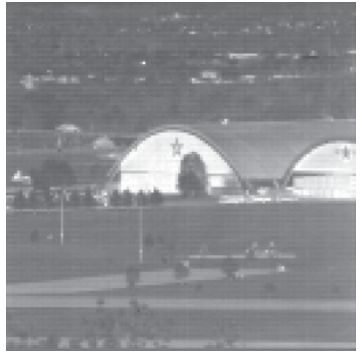

(b)

(d)

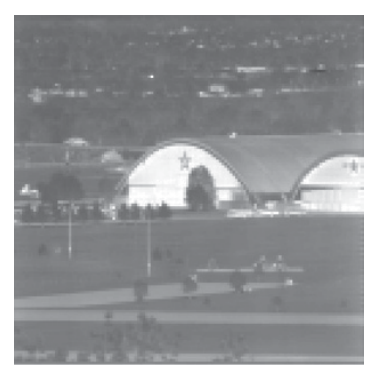

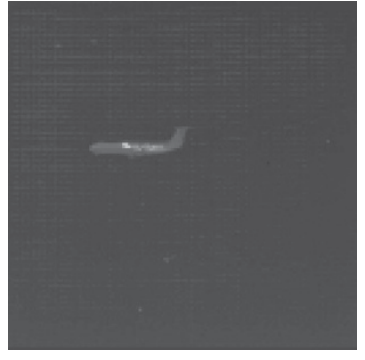

(a)

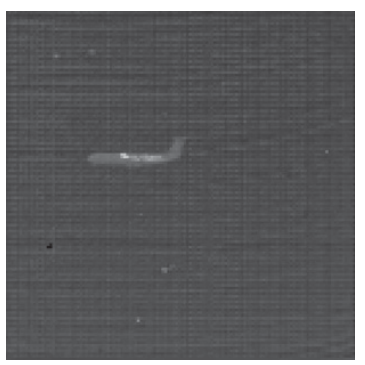

(c)

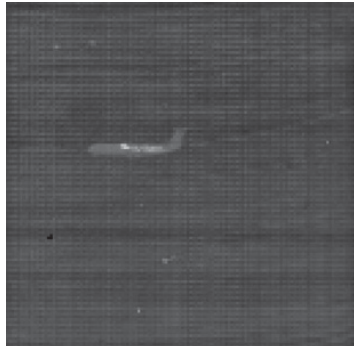

(b)

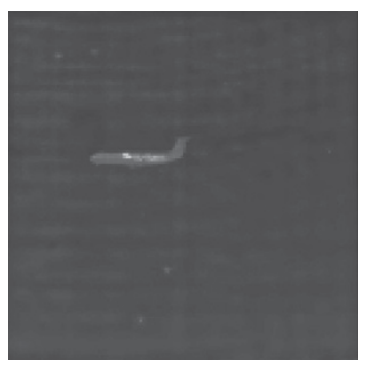

(d)
Figure 8: Adaptive NUC results on real infrared data sequence taken at 9:30 AM. (a) Real infrared data $(\rho=0.0928)$. (b) NUC with $\eta=0.001$ and a $21 \times 21$ averaging window $(\rho=0.0613)$. (c) NUC with $\eta=0.0025$ and a $3 \times 3$ averaging window $(\rho=0.0539)$. (d) NUC with a $3 \times 3$ averaging window with adaptive learning rate using $k_{\text {alr }}=0.075(\rho=0.0519)$.

information for calculating the adaptive learning rate rule, in order to improve even more the performance and robustness of the new technique.

\section{ACKNOWLEDGMENTS}

The authors thank Ernest E. Armstrong, Stephen C. Cain, Majeed M. Hayat, and the US Air Force Research Laboratory (Dayton, Ohio) for their valuable suggestions and assistance. This work was supported by the Chilean National Foundation for Science and Technology (FONDECYT) projects nos. 1020433 and 7020433. Sergio Torres also thanks the Milenio Grant ICM P02-049 of the Chilean Government.

\section{REFERENCES}

[1] D. Scribner, M. Kruer, and J. Killiany, "Infrared focal plane array technology," Proc. IEEE, vol. 79, no. 1, pp. 66-85, 1991.

[2] G. Holst, CCD Arrays, Cameras and Displays, SPIE Optical Engineering Press, Bellingham, Wash, USA, 1996.

[3] R. Driggers, P. Cox, and T. Edwards, Introduction to Infrared and Electro-Optical Systems, Artech House Publishers, Boston, Mass, USA, 1999.

[4] A. Friedenberg and I. Goldblatt, "Nonuniformity two-point linear correction errors in infrared focal plane arrays," Optical Engineering, vol. 37, no. 4, pp. 1251-1253, 1998.

[5] P. Narendra, "Reference-free nonuniformity compensation for IR imaging arrays," in Smart Sensors II, vol. 252 of Proceedings of SPIE, pp. 10-17, San Diego, Calif, USA, JulyAugust 1980.
Figure 9: Adaptive NUC results on real infrared data sequence taken at an unknown time. (a) Real infrared data $(\rho=0.0188)$. (b) NUC with $\eta=0.001$ and a $21 \times 21$ averaging window ( $\rho=$ 0.0535 ). (c) NUC with $\eta=0.0025$ and a $3 \times 3$ averaging window $(\rho=0.0452)$. (d) NUC with a $3 \times 3$ averaging window with adaptive learning rate using $k_{\mathrm{alr}}=0.075(\rho=0.0373)$.

[6] J. G. Harris and Y.-M. Chiang, "Nonuniformity correction of infrared image sequences using the constant-statistics constraint," IEEE Trans. Image Processing, vol. 8, no. 8, pp. 11481151, 1999.

[7] M. Hayat, S. Torres, E. Armstrong, S. Cain, and B. Yasuda, "Statistical algorithm for nonuniformity correction in focalplane arrays," Applied Optics, vol. 38, no. 5, pp. 772-780, 1999.

[8] R. Hardie, M. Hayat, E. Armstrong, and B. Yasuda, "Scenebased nonuniformity correction with video sequences and registration," Applied Optics, vol. 39, no. 8, pp. 1241-1250, 2000.

[9] S. Cain, M. Hayat, and E. Armstrong, "Projection-based image registration in the presence of fixed-pattern noise," IEEE Trans. Image Processing, vol. 10, no. 12, pp. 1860-1872, 2001.

[10] B. Ratliff, M. Hayat, and R. Hardie, "An algebraic algorithm for nonuniformity correction in focal-plane arrays," Journal of the Optical Society of America $\{A\}$, vol. 19, no. 9, pp. 17371747, 2002.

[11] S. Torres and M. Hayat, "Kalman filtering for adaptive nonuniformity correction in infrared focal-plane arrays," Journal of the Optical Society of America $\{A\}$, vol. 20, no. 3, pp. 470-480, 2003.

[12] S. Torres, J. Pezoa, and M. Hayat, "Scene-based nonuniformity correction for focal plane arrays by the method of the inverse covariance form," Applied Optics, vol. 42, no. 29, pp. 5872-5881, 2003.

[13] D. Scribner, K. Sarkady, M. Kruer, et al., "Adaptive nonuniformity correction for IR focal-plane arrays using neural networks," in Infrared Sensors: Detectors, Electronics and Signal Processing, vol. 1541 of Proceedings of SPIE, pp. 100-109, San Diego, Calif, USA, July 1991. 
[14] D. Scribner, K. Sarkady, M. Kruer, et al., "Adaptive retina-like preprocessing for imaging detector arrays," in Proc. IEEE International Conference on Neural Networks (ICNN '93), vol. 3, pp. 1955-1960, San Francisco, Calif, USA, February 1993.

[15] B. Widrow and S. Stearns, Adaptive Signal Processing, Prentice Hall, Englewood Cliffs, NJ, USA, 1985.

[16] J. Principe, N. Euliano, and W. C. Lefebvre, Neural and Adaptive Systems: Fundamentals through Simulations, John Wiley \& Sons, New York, NY, USA, 2000.

Esteban Vera was born in Santiago, Chile, in 1975. He received his B.S. degree in electronic engineering at the University of Concepcion, Chile, in 1999. He received his M.S. degree in electrical engineering from the University of Concepcion, Chile, in 2003. He has developed his professional carreer in the astronomy field, having worked for several large telescope projects, first at the European Southern Observatory and now at

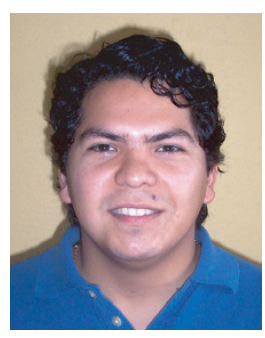
the Gemini Observatory, where he is a specialist in infrared and ccd detectors for scientifical instrumentation. His research interests are in digital signal and image processing, focused on the application of wavelets, neural networks, and independent component analysis for solving problems such as blind source separation and superresolution.

Sergio Torres was born in Concepcion, Chile, in 1962. He received the B.S.E.E. degree from the University of Concepcion, Chile, in 1987. He obtained the M.S. and the $\mathrm{Ph} . \mathrm{D}$. degrees in electrical engineering from the University of Dayton, Dayton, Ohio, USA, in 1997 and 2001, respectively. From 1987 to 1994, he was a Faculty Member of the Electrical Engineering Department, the University of Concepcion, Chile. From 1994

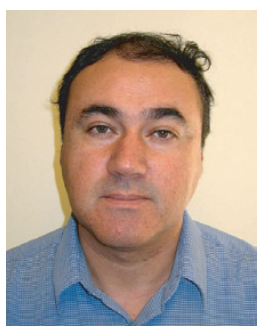
to 2001, he was a graduate student at the University of Dayton, Ohio, USA and worked as a Research Assistant in projects founded by the NSF and the US Air Force Research Laboratory. He now joints the Electrical Engineering Department, the University of Concepcion, Chile, as an Associate Professor. His areas of interest are digital signal processing applied to electro-optical systems, optical sensors, and optical communications. 